\title{
Physical Activity With Tailored mHealth Support for Individuals With Intellectual Disabilities: Protocol for a Randomized Controlled Trial
}

Henriette Michalsen ${ }^{1,2}$, PsyD; Silje Camilla Wangberg ${ }^{3}$, PsyD, PhD; Gunnar Hartvigsen ${ }^{4}$, MSc, PhD; Letizia Jaccheri ${ }^{5}$, MSc, PhD; Miroslav Muzny ${ }^{1}$, MSc; André Henriksen ${ }^{6}$, MSc, MBA; Monica Isabel Olsen ${ }^{1,2}$, MSc; Gyrd Thrane ${ }^{7}$, $\mathrm{PhD}$; Reidun Birgitta Jahnsen ${ }^{8,9}, \mathrm{PhD}$; Gunn Pettersen ${ }^{7}, \mathrm{PhD}$; Cathrine Arntzen ${ }^{1,7}, \mathrm{PhD}$; Audny Anke ${ }^{1,2,9}, \mathrm{MD}, \mathrm{PhD}$

${ }^{1}$ Department of Rehabilitation, University Hospital of North Norway, Troms $\varnothing$, Norway

${ }^{2}$ Department of Clinical Medicine, University of Troms $\emptyset$ - The Arctic University of Norway, Troms $\emptyset$, Norway

${ }^{3}$ Department of Health and Care Sciences, University of Troms $\varnothing$ - The Arctic University of Norway, Narvik, Norway

${ }^{4}$ Department of Computer Science, University of Troms $\varnothing$ - The Artic University of Norway, Troms $\varnothing$, Norway

${ }^{5}$ Department of Computer Science, The Norwegian University of Science and Technology, Trondheim, Norway

${ }^{6}$ Department of Community Medicine, University of Troms $\varnothing$ - The Arctic University of Norway, Troms $\varnothing$, Norway

${ }^{7}$ Department of Health and Care Sciences, University of Troms $\varnothing$ - The Arctic University of Norway, Troms $\varnothing$, Norway

${ }^{8}$ Department of Neurosciences for Children, Oslo University Hospital, Oslo, Norway

${ }^{9}$ Research Centre for Habilitation and Rehabilitation Models and Services, University of Oslo, Oslo, Norway

\section{Corresponding Author:}

Henriette Michalsen, PsyD

Department of Rehabilitation

University Hospital of North Norway

Hansine Hansens vei 67

Tromsø, 9038

Norway

Phone: 4741500304

Email: henriette.michalsen@uit.no

\begin{abstract}
Background: Individuals with intellectual disabilities (IDs) have lower levels of physical activity (PA) and greater barriers for participation in fitness activities compared with members of the general population. As increased PA has positive effects on cardiovascular and psychosocial health, it is exceedingly important to identify effective interventions for use in everyday settings. Mobile health (mHealth) methods such as motion sensor games (exergames) and smartphone reminders for PA have been explored and found to be promising in individuals with IDs.
\end{abstract}

Objective: The purpose of this study is to examine the effectiveness of an individually tailored PA program with motivational mHealth support on daily levels of PA in youth and adults with IDs.

Methods: The trial uses a randomized controlled design comprising 30 intervention participants and 30 control group participants, aged 16 to 60 years, with sedentary lifestyles or low PA levels. While the controls will receive standard care, the intervention aims to increase the level of PA, measured as steps per day, as the primary outcome. Secondary outcome variables are body mass index, blood pressure, physical performance, social support for PA, self-efficacy in a PA setting, behavior problems, and goal attainment. The intervention involves the delivery of tailored mHealth support, using smartphones or tablets to create structure with focus on the communicative abilities of individual participants. Rewards and feedback are provided in order to motivate individuals to increase participation in PA. Participants in the intervention group, their close relatives, and care staff will be invited to participate in a preintervention goal-setting meeting, where goal attainment scaling will be used to select the participants' PA goals for the intervention period. All participants will be assessed at baseline, at 3 months, and at 6 months.

Results: Enrollment was planned to start in April 2020 but will be delayed due to the pandemic situation. The main contribution of this paper is a detailed plan to run our study, which will produce new knowledge about tailored mHealth to support PA in individuals with intellectual disabilities. 
Conclusions: We expect the new intervention to perform better than standard care in terms of improved PA, improved self-efficacy, and social support for activities. Technology offers new opportunities to promote healthy behaviors. The results of the study will determine the effectiveness and sustainability of a tailored mHealth support intervention to increase PA in youth and adults with IDs.

Trial Registration: ClinicalTrials.gov NCT04079439; https://clinicaltrials.gov/ct2/show/NCT04079439

International Registered Report Identifier (IRRID): PRR1-10.2196/19213

(JMIR Res Protoc 2020;9(6):e19213) doi: 10.2196/19213

\section{KEYWORDS}

intellectual disability; physical activity; technology; mHealth; mobile phone; goal attainment; social support; self-efficacy

\section{Introduction}

The prevalence of intellectual disabilities (IDs) is estimated to be $1 \%$ of the world's population [1,2]. Compared with the general population, individuals with IDs have worse health and lower levels of activity [3-5], and they have greater barriers for participating in fitness activities [6] and accessing health care services $[7,8]$.

\section{Physical Activity Guidelines}

The World Health Organization (WHO) guidelines for physical activity (PA) state that typical adults should spend a minimum of 150 minutes per week engaged in moderate-intensity PA or 75 minutes engaged in vigorous-intensity PA [9]. One systematic review found that only $9 \%$ of individuals with IDs met the WHO's minimum PA guidelines [4]. Norwegian guidelines are in line with the international guidelines and recommend 150 minutes of moderate- to vigorous-intensity PA per week for adults [10] and 60 minutes per day for children and youths [11]. As high PA is a determinant of health and increased activity has positive effects on cardiovascular and psychosocial health, identifying effective interventions for use in everyday settings is exceedingly important.

\section{Physical Activity Interventions}

Some well-designed studies have not been able to demonstrate improved levels of PA in intervention groups of individuals with IDs after the intervention period has ended. One theory-based randomized controlled study of adults with all types of IDs did not find any significant increases in levels of PA (steps per day) using a walking program [12]. Furthermore, the results of a cluster-randomized study of older adults in the Netherlands showed marginal effects and substantial missing data, despite being well prepared with a published protocol and using day-activity centers for the intervention [13]. Past controlled studies on the effects of PA interventions on individuals with IDs have primarily included adults with mild to moderate IDs, and effect sizes have been small [5,14]. Some studies have reported improved effects on physical fitness indicators such as balance and muscle strength [15], psychological well-being [16], perception of social competence [17], and work routines [14] after increasing levels of PA. One recent study included individuals with severe or profound IDs in a technology-aided program for promotion of PA and found positive results in energy expenditure and independent engagement in light to moderate PA [18], but with a small number of participants. Findings from previous studies indicate that a more flexible approach [19], greater use of theory in intervention design, stronger research design (as there are few randomized controlled studies), and better translation of interventions to community-based settings [20] are needed. Future studies could also have an ecological approach, where the interplay between individual, interpersonal, and environmental factors are considered [1,21]. Motivational issues have been challenging, particularly for approaches that aim for sustainable effects [17], and should be considered when designing future PA interventions.

\section{Mobile Health Interventions}

Mobile health (mHealth) provides a wide range of possibilities for monitoring and motivating individuals in the self-management of chronic illnesses [22-24]. Motion sensor games (exergames) have been explored and have been found to be promising in individuals with IDs [25]. For these solutions to move out of the lab and into actual use, they need to first meet users' needs [26]. The mobile platform is ubiquitous, and the touch interface has proven to have a low level of cognitive demand and could be used to improve adherence to PA [27]. At present, few mobile apps have incorporated games strategies, such as goal setting or rewards, to increase PA in individuals with various disabilities [28]. To our knowledge, there has been only 1 preliminary report (letter) of a randomized controlled trial using smartphone support to increase motivation for PA in youth and adults with IDs [29].

Methods that could facilitate the development of individualized mHealth support solutions include tailoring, individual goal setting [30], and adjusting communication to meet the specific needs of individuals with IDs [31]. Studies on motivation for PA in the IDs population have shown that predictability with routine and familiarity, communication of purpose, and enjoyable and social activities promote motivation and participation [21,32]. Family and care staff involvement will be central, and we expect the study's implementation in a natural setting to enhance the effect [33]. We also expect the systematic use of mHealth with rewards and gamification to be beneficial. In Norway, many individuals with IDs have a smartphone or a tablet that they can use for tailored PA interventions. However, this use has not been tested previously. We expect a motivational app for smartphones and tablets to promote adherence to PA in individuals with IDs. According to the World Report on Disability, health promotion efforts targeting this population can improve lifestyle behaviors [34]. The report states that these individuals have the right to be included in all PA programs. 
Thereby, the present study aims to examine the effectiveness of an individually tailored PA program with motivational mHealth support on everyday levels of PA in youth and adults with IDs, targeting individual, contextual, and interactional factors of PA participation [21]. In addition to higher levels of PA, we expect improvements in psychological health, self-efficacy in activities [32], and social support for physical activity participation [35].

\section{Methods}

\section{Design}

The current study has a randomized controlled clinical design with assessments at baseline, 3 months, and 6 months. Participants will receive either the tailored mHealth-supported PA program or standard care with access to the mHealth support system once the trial is completed. Family and care staff will be involved in the program for support and follow-up.

\section{Participants}

A total of 60 participants will be recruited into the trial through health care and other related organizations in the municipality of Troms $\varnothing$ in northern Norway. Information about the study will be delivered at meeting places, such as day and activity centers. If the number of included participants is insufficient, more municipalities in Northern Norway will be included, or a multicenter approach will be considered.

\section{Inclusion and Exclusion Criteria}

Individuals will be included if they have a sedentary lifestyle [1] or a low level of PA, determined with the question, "In how much of your leisure time have you been physically active in the last year?" [8]. The question has 4 response categories and has been used in population-based studies of the general population [36] and in European health indicator studies of individuals with IDs [8]. Individuals with a sedentary lifestyle (eg, primarily engaged in reading, watching television, or other mainly sedentary activities) and a low level of PA (eg, engaged in walking or other light PA for less than 4 hours a week) will be included in the study. Other inclusion criteria will be as follows: (1) diagnosis of ID (mild, moderate, severe, or profound), (2) aged between 16 and 60 years, (3) ability to participate in the intervention, (4) ability to walk with or without support, and (5) able to provide written informed consent or written informed consent can be obtained from a representative. Prior to enrollment, all participants will be screened for readiness, and, if necessary, medical clearance will be obtained. The Physical Activity Readiness Questionnaire [37] will be used for this purpose. Exclusion criteria will be as follows: (1) medical contraindications for participation in programs with increased exercise as advised by the primary care or ID specialist physician, (2) high level of physical activity, and (3) inability to provide written informed consent and written informed consent cannot be obtained from a representative.

\section{Ethics}

This study has been approved by the Regional Committee for Medical and Health Research Ethics in northern Norway (number 2016/1770). The protocol has been registered at
ClinicalTrials.gov under the identifier NCT04079439. The project adheres to the Consolidated Standards of Reporting Trials guidelines. Written informed consent will be obtained from each participant, their legal representative, or both prior to inclusion in the study and baseline assessment. If the participant has impaired capability to consent, consent will be sought from the nearest relative or guardian as well as from the individual with IDs, or as representative consent. Ethical issues will be continuously considered. Any adverse events will be recorded.

\section{Randomization}

Participants will be randomized with a computer program to either the PA intervention with mHealth support group or the standard care control group.

\section{Intervention Group}

This randomized controlled trial is part of our project to develop a tailored mHealth support system that promotes PA in individuals with IDs [38]. In previous parts of the project, we conducted a qualitative study on motivation for participation in PA for individuals with IDs [21], held workshops and collaborated with mHealth developers, and performed usability tests. This process is illustrated in Figure 1.

Findings from the qualitative study, discussions in workshops, and creative meetings among developers and researchers showed that many individuals with IDs experience difficulties participating in PA because of individual, interactional, and contextual factors. Some of these factors include individual difficulties in initiating activities; preferences for fun and social activities; and lack of social support, availability of activities, resources and preparation, predictability, and collaboration in activities. After examination of these findings, a prototype of an app was created and presented in one of the workshops. The feedback was promising, and development of the mHealth support system continued. The main emphasis in the app is individually chosen activities, tailored communication, predictability, use of rewards, and involvement of support persons. Activities will be chosen during a goal attainment meeting (using goal attainment scaling) [39] with the individual with ID and a support person (usually a family member or health care provider). Goal attainment is widely used as an outcome measure within rehabilitation medicine [39] and has been used in studies with individuals with IDs [40]. The research group is familiar with the use of goalsetting processes in previous studies with individuals with IDs. The final intervention will consist of an advanced activity planner based on the platform for the app Active Leisure (Smart Cognition AS). Actiplan features and tracks daily physical activities. The app offers different interface options (symbols only, easy-to-read text, plain text, and read aloud). See Figure 2 for examples. The activity planner will mostly be used by the individual with ID and a support person (caregiver or health care provider). After completing an activity, a simple reward is available (eg, a smiling face or sharing a picture with other users of the app). The use of tailored communication [41] is available through personalization, including the use of the individual's first name in the activity planner, individually chosen activities, adjusted communication (eg, symbols, easy-to-read text, or plain text), 
preparation and planning, and feedback. Rewards and positive feedback after activities have been performed are expected to increase motivation, and thereby lead to higher levels of PA.

In addition, 3 individual exercise apps have been developed as potential alternatives that can be added to Actiplan: (1) an exergame or game-inspired app that promotes outdoor PA; (2) an ergometer bike or outdoor bike placed inside the home, with a mounted screen showing a video or other visually interesting features; and (3) a game-inspired, avatar-based health platform for monitoring PA and motivating users to increase their PA levels [38].

To explore the participants' expectations and experiences of the intervention and the mHealth support, a qualitative pilot study recruiting 10 of the first participants in the intervention group will be performed.

Figure 1. Development process of the electronic health support component of the study.

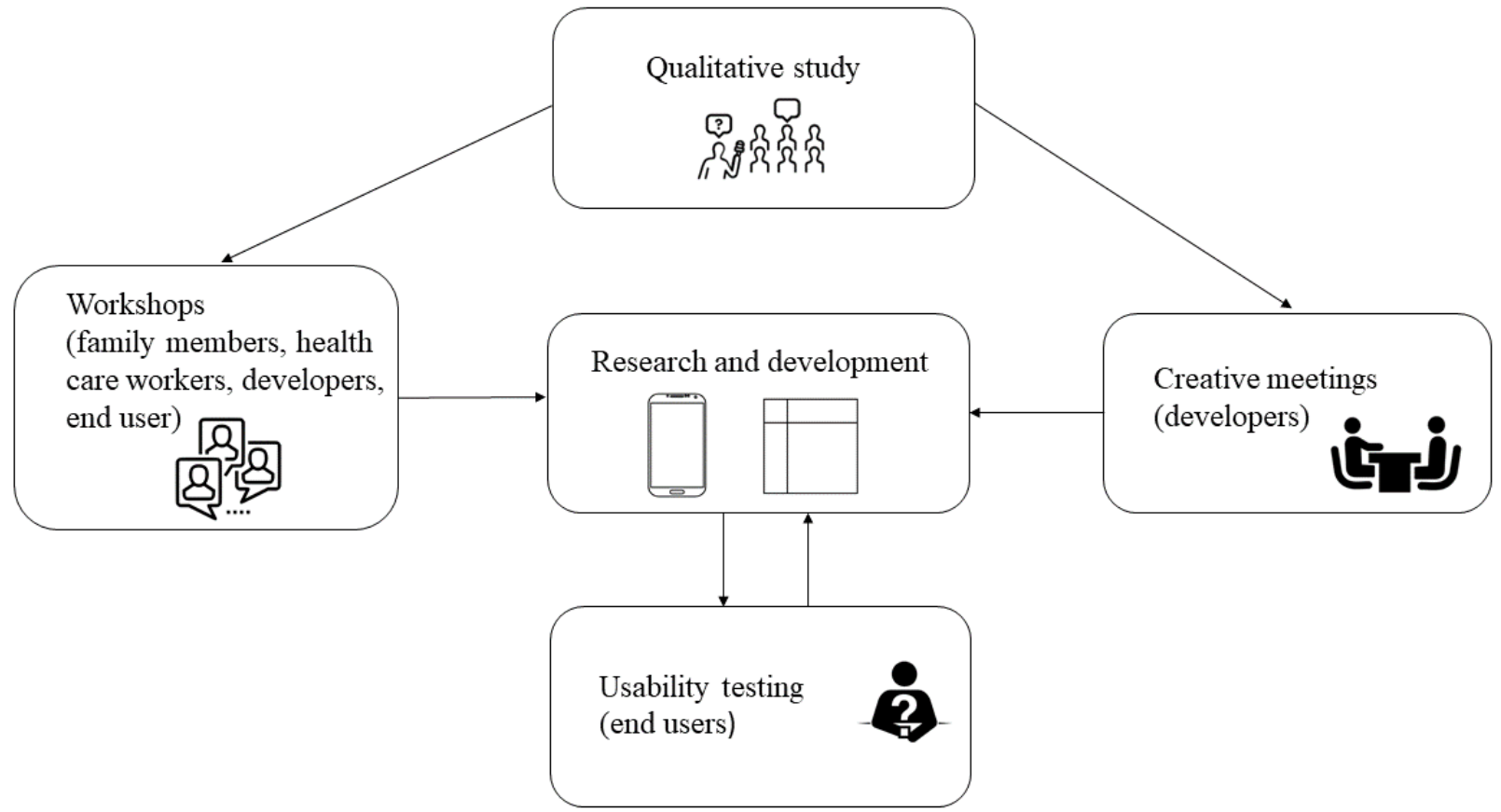

Figure 2. Interface options of the Actiplan app: symbols only, easy-to-read text, or plain text. The app also has read-aloud capabilities.
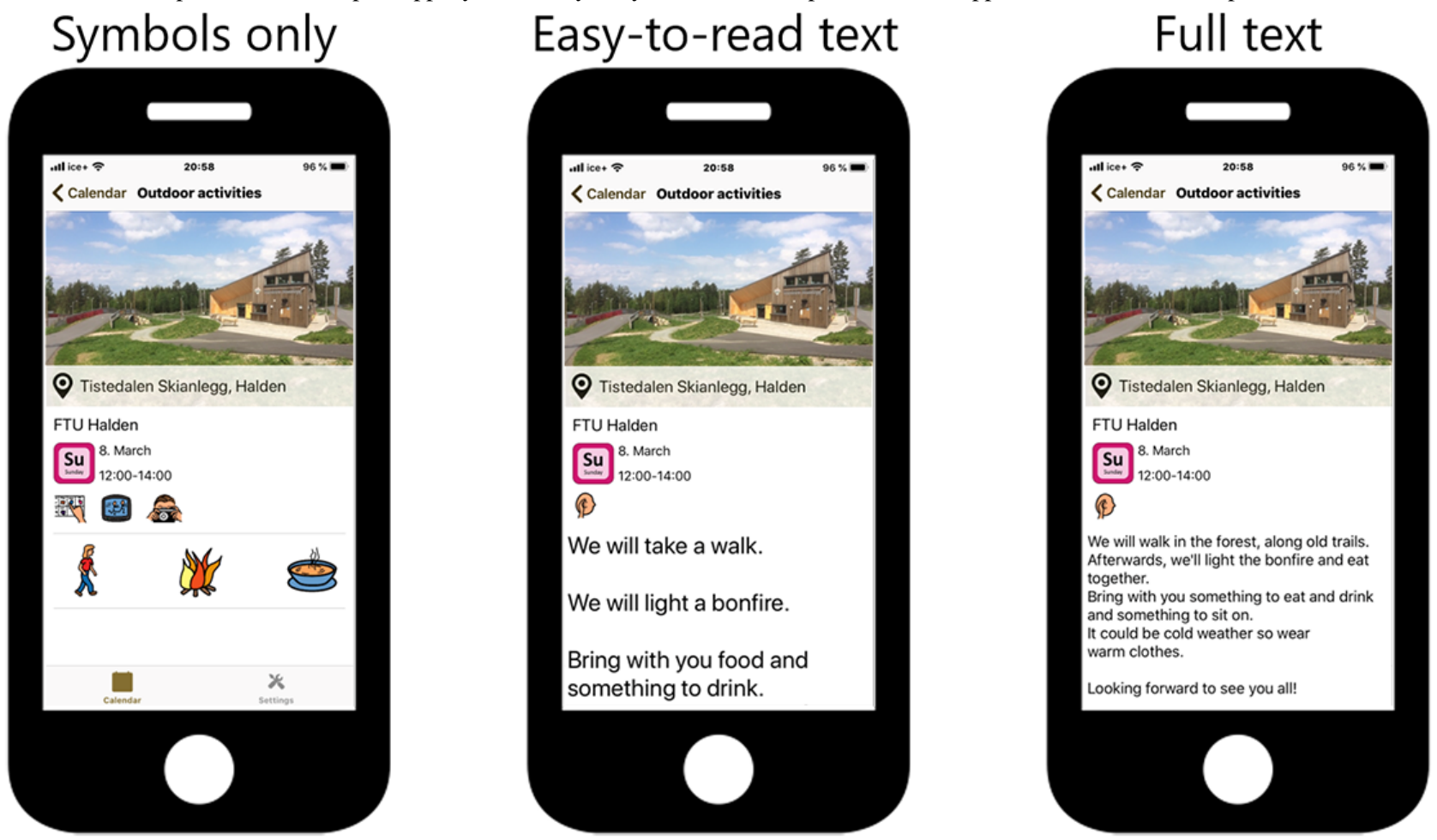


\section{Control Group}

Participants in the control group will be administered the assessments at baseline, 3 months, and 6 months, and otherwise continue with their standard everyday activities during the study period. They will be invited to use the mHealth support system at the end of the 6-month intervention period.

\section{Data Collection}

Data will be collected at baseline, 3 months, and 6 months, as seen in Figure 3. Baseline data will include baseline PA activity level and will be collected before randomization. Follow-up data will be collected regardless of the participant's compliance with the study protocol. Participants and assessors will not be blinded. Background data on the participants will be collected at baseline and will include age, gender, educational level, marital status, living condition, employment status, educational status, job-related or day center activities, leisure time activities, smoking habits, level of ID (ie, mild, moderate, severe, or profound), genetic diagnosis, gross motor function classification $[42,43]$, communication level [44], medical history/readiness for the PA intervention, and use of medication. In addition, we will ask questions about barriers for participation in physical activities.

Figure 3. Consolidated Standards of Reporting Trials flow diagram of the study. mHealth: mobile health.

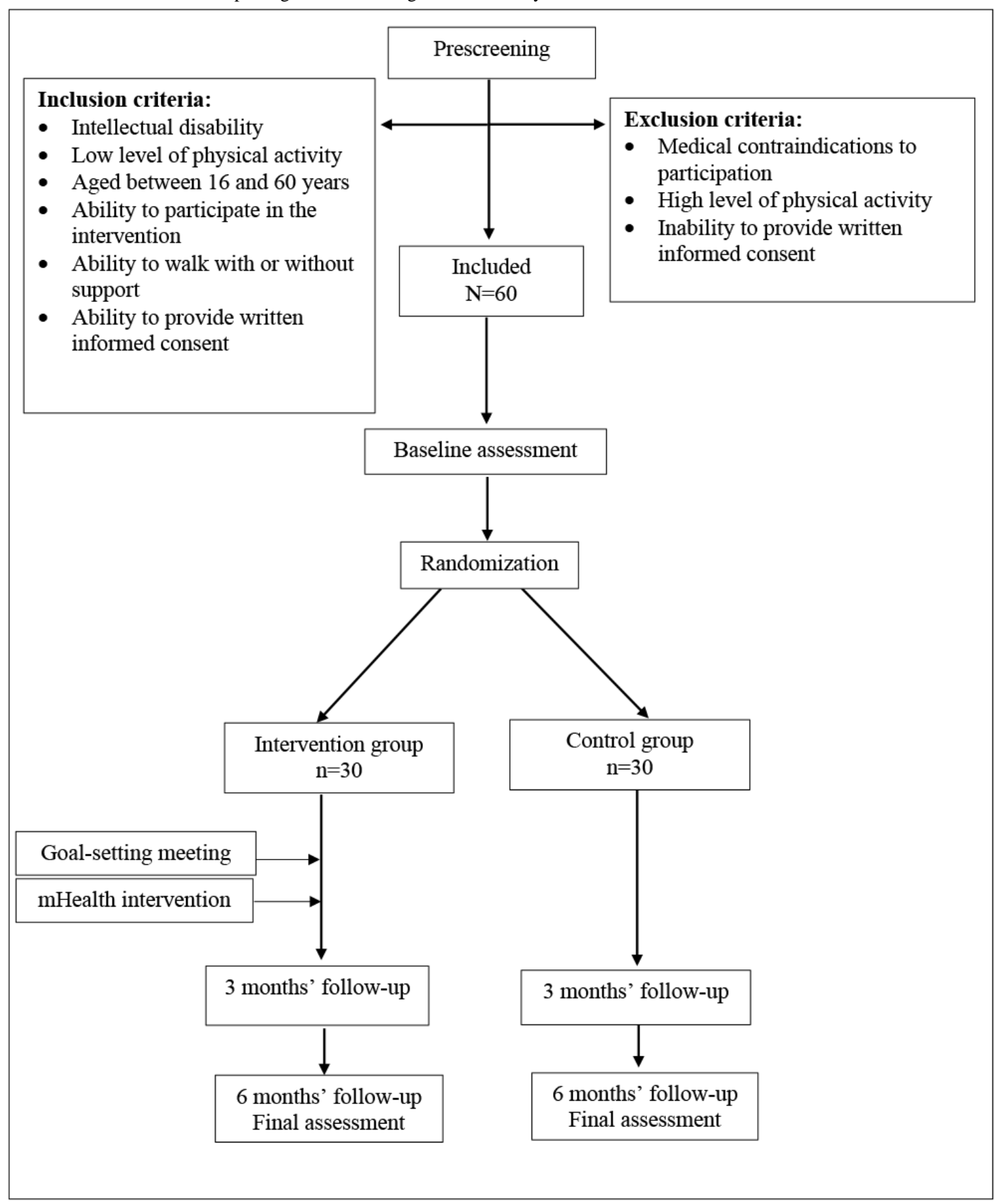




\section{Outcome Measures}

\section{Primary Outcome}

The primary outcome of this study will be objectively measured PA, as assessed by steps per day measured with a wrist-worn commercial accelerometer (Fitbit Versa; Fitbit Inc). The device will assess level of PA and sedentary time [45]. The watch will be covered (neutral screen) during baseline and follow-up assessments in both the intervention and control groups. Screen neutrality is achieved by running a custom app on the watch that disables all buttons and prevents screen feedback (except showing current time). This app cannot be disabled by the participant. Level of PA will be measured for 7 days at each assessment, with a minimum of 3 consecutive days of measurement because previous research has shown that 3 days of PA can predict the weekly level of PA [14,46]. Many of the commercial fitness trackers have been validated for use in research [47], including devices from Fitbit Inc [48,49].

\section{Secondary Outcomes}

Secondary outcome measures will include minutes of moderate PA per day, PA questionnaire, body mass index, blood pressure, physical performance, social support for physical activity, self-efficacy in a PA setting, behavior problems, and goal attainment. See Table 1 for a summary of all outcome measures.

Table 1. Summary of outcome measures.

\begin{tabular}{lll}
\hline Measurement & Type of outcome & Measure \\
\hline Steps per day & Primary outcome & Fitness tracker \\
Minutes of moderate activity & Secondary outcome & Fitness tracker \\
Body mass index & Secondary outcome & $\mathrm{kg} / \mathrm{m}^{2}$ \\
Blood pressure & Secondary outcome & Blood pressure monitor \\
Physical performance & Secondary outcome & Short Physical Performance Battery [50] \\
Behavior problems & Secondary outcome & Aberrant Behavior Checklist-Community [51] \\
Social support for PA and self-efficacy in PA setting & Secondary outcome & The Self-Efficacy/Social Support for Activity for \\
& & Persons With Intellectual Disability scale [35] \\
Goal attainment & Method, secondary outcome & Goal attainment scaling [39] \\
Satisfaction with life & Control for adverse effects & Satisfaction with life scale [52]
\end{tabular}

\section{Physical Activity}

The secondary PA outcome is the number of minutes of moderate PA per day, measured with the commercial accelerometer.

In addition, the International Physical Activity Questionnaire-Short Form, adapted to measure PA using proxy respondents, will be used [53]. The International Physical Activity Questionnaire-Short Form is a 7-item questionnaire that assesses PA in the past 7 days at 4 intensity levels: (1) vigorous-intensity activity, such as aerobics, (2) moderate-intensity activity, such as leisure cycling, (3) walking, and (4) sitting.

\section{Body Mass Index and Blood Pressure}

Body mass index will be calculated in $\mathrm{kg} / \mathrm{m}^{2}$ [54]. Blood pressure will be measured using a blood pressure monitor (Welch Allyn Inc). Height will be measured in meters with a stadiometer (Seca $\mathrm{GmbH}$ ), with the participant wearing no shoes. Weight will be measured in kilograms with an analog floor scale (Seca $\mathrm{GmbH}$ ), with participants wearing no shoes or outdoor jackets/gear. Waist circumference will be measured $1 \mathrm{~cm}$ above the navel.

\section{Physical Performance}

The Short Physical Performance Battery (SPPB) will be used to assess physical performance. The SPPB is a screening test designed to evaluate physical performance and predict disability in older adult populations [50]. The SPPB is mainly a measure of lower-extremity function and consists of 3 subtests: static balance, gait speed, and lower limb strength. To measure static balance, the participant is asked to stand with feet in the side-by-side, semitandem, and tandem positions, for 10 seconds each, to his or her best ability. Gait speed is measured with a 4-m (13-ft) walk at the individual's habitual pace. Lower limb strength is measured by having the participant rise from a chair with arms folded across his or her chest, to his or her best ability. Subtest scores range from 0 (inability perform the test) to 4 (highest level of performance). The SPPB has been validated [55], and reference values have been published [56]. The Norwegian version of the SPPB appears to have acceptable reliability as well as good internal consistency in an older population with and without dementia [57]. The SPPB has been used in people with mild and moderate IDs $[58,59]$.

\section{Behavior Problems}

The Aberrant Behavior Checklist-Community (ABC-C) is a questionnaire designed to assess challenging behavior in children, youth, and adults with IDs [51]. The checklist consists of 58 items divided into 5 subscales: irritability, lethargy, stereotypy, hyperactivity, and inappropriate speech. It is a proxy measure requiring knowledge of the person with ID. Each item is scored on a scale of 0 to 3 ( 3 indicating the highest severity). The ABC-C is a widely used behavioral rating scale used among people with IDs. A Norwegian study [60] examined the construct validity of the Norwegian $\mathrm{ABC}$ in a clinical sample of children and youths in Norway and found satisfactory psychometric properties for the $\mathrm{ABC}$, with the exception of the 
inappropriate speech factor. The Cronbach coefficients were adequate to excellent, with coefficients ranging from .76 to .95 . The ABC subscales were moderately to highly correlated with one another $(r=0.41-0.78, P<.001)$.

\section{Social Support for Physical Activity and Self-Efficacy in a PA Setting}

The Self-Efficacy/Social Support for Activity for Persons with Intellectual Disability scale [35] is a questionnaire consisting of 4 subscales. One subscale measures self-efficacy for overcoming barriers to leisure PA. The last 3 subscales measure social support for leisure activity from family members, care staff, and friends for individuals with IDs. The scale has been validated for self-reporting from individuals with mild to moderate IDs and for use by proxy respondents [35]. The questionnaire will be translated into the Norwegian language using standard guidelines [61].

\section{Goal Attainment}

Goal attainment scaling $[39,62]$ will be used to identify self-management goals that participants would like to achieve. The questionnaire will be filled out by the researcher, with participants and proxy respondents present. Goal attainment scaling involves several steps. Goals are selected by each individual, and observable behavior that reflects a degree of goal attainment is defined [63]. The participant's pretreatment or baseline levels are defined in terms of the goal. Five different goal attainment levels are used, ranging from "no change" to "much better than expected outcome" (numbered -2 to +2 ). Follow-up times for participant evaluation are set after 3 months and 6 months. Goal attainment is evaluated after the defined time interval. At the end, the overall attainment score for all goals are calculated. In this study we will define up to 3 goals for PA [64]. The scale has been validated for use in rehabilitations populations [39] and has been used in studies with individuals with IDs $[40,65]$.

\section{Satisfaction With Life}

This study will use the satisfaction with life scale developed by Bergström and Hochwälder [52], which was designed to assess satisfaction with the home environment and leisure time in individuals with mild to moderate IDs. The scale has 4 factors: (1) satisfaction with housing environment, (2) satisfaction with life, (3) satisfaction with meals, and (4) satisfaction with recreational activities. Items are read aloud by a researcher and answered by participants with 3 response options: good (happy face $=2$ ), in between (neutral face $=1$ ), or bad ( $\operatorname{sad}$ face $=0$ ). In the current study, the scale is used to control for adverse effects.

\section{Data Integrity}

Patient- or proxy-reported and assessor-reported data are partly captured electronically using Research Electronic Data Capture (REDCap) (Vanderbilt University). REDCap is a web-based system that is compliant with relevant regulations and security requirements. The system has an integrated function for randomization. Questionnaires can be sent electronically to participants or the proxy respondent. Data not captured electronically, such as background information and physical performance test results, are registered at the baseline meeting. The study coordinator will evaluate the data of all participants for completeness. In cases of missing data, respondents will be contacted.

\section{Statistical Analyses}

\section{Sample Size}

The study will be powered based on the primary outcome of accelerometer-measured steps per day (mean of 4 days) $[4,66]$. With a 2-group design and effect size of 0.8 , power of $80 \%$, and of .05 , the expected minimum total sample size is 50 participants ( 25 participants in each group). The effect size in the current study is estimated based on previous studies, which have reported Cohen $d$ values ranging from 0.29 to 0.91 $[14,37,67]$. An effect size of 0.8 is considered to be a clinically relevant difference between the 2 groups, corresponding to an increase in steps per day of approximately 2000 in the intervention group, which is also expected to be achievable [14]. To avoid underpowering the study and to prepare for expected dropout, we will recruit 30 participants per group, for a total sample size of 60 (Figure 3 ). The group size estimated is supported by other randomized controlled study protocols $[27,37]$ and published results [68] of studies to enhance levels of physical activity in individuals with ID.

\section{Data Analyses}

The randomized controlled trial includes repeated measures in 2 groups, and linear mixed models will be used in the efficacy analyses of the intervention. In addition to a group variable (treatment or control), follow-up time (3 months and 6 months) and mean steps (with baseline comparison) will be added as covariates. An intention-to-treat approach will be used with a significance level of $P<.05$ and a secondary per-protocol analysis. All analyses will be performed using SPSS 26 software (IBM Corp).

\section{Results}

The project is approved by the Regional Committee for Medical and Health Research Ethics in northern Norway and is registered at ClinicalTrials.gov. Enrollment was planned to start in April 2020 but will be delayed due to the pandemic situation. Participant recruitment for the randomized controlled study will be initiated as soon as practical difficulties due to the pandemic situation are solved. Participants will be recruited, randomized, and administered the intervention individually. The main contribution of this paper is a detailed plan to run our study, which will produce new knowledge about mHealth to support PA in individuals with IDs.

\section{Discussion}

The present trial will investigate how modern technology and mHealth can be used in the promotion of PA in individuals with IDs. A tailored PA program is expected to increase levels of PA, and individuals with IDs and low PA have the greatest chances of improving [13]. Throughout our project, we have used an ecological approach, and we are currently developing a theory-based mHealth motivational support system for the promotion of PA, which we believe will increase the probability of improved levels of PA. Focusing on the communication 
abilities of each participant and individual goal setting may be particularly important [64]. As previous research has shown difficulties in recruitment and data collection, including missing follow-up data [13], we will be prepared to meet these challenges.

By including individuals with all types of IDs and low levels of PA, we can add to the knowledge on whether mHealth support can be successfully adjusted to individuals with different levels of functioning and whether it can increase levels of PA
[5]. There is evidence demonstrating that an mHealth intervention for PA can improve self-efficacy in activities, social support [32], health conditions such as blood pressure [13], and the results of physical performance tests [69]. This study has potentially important implications for both individuals with IDs and their support networks. If successful, the project will provide a simple and accessible solution for promoting PA in individuals with IDs. For widespread clinical implementation, it is necessary to engage stakeholders who support individuals with IDs throughout their lives.

\section{Acknowledgments}

The study has been and will continue to be conducted with grants from the Northern Norway Regional Health Authority (grant number HNF1353-17) and has received and will continue to receive support from the Department of Clinical Research, University Hospital of North Norway, Troms $\emptyset$, Norway.

\section{Conflicts of Interest}

None declared.

\section{References}

1. Oppewal A, Hilgenkamp T, Schäfer Elinder L, Freiberger E, Rintala P, Guerra-Balic M, et al. Correlates of Sedentary Behaviour in Adults with Intellectual Disabilities-A Systematic Review. Int J Environ Res Public Health 2018 Oct 17;15(10) [FREE Full text] [doi: 10.3390/ijerph15102274] [Medline: 30336554]

2. Maulik PK, Mascarenhas MN, Mathers CD, Dua T, Saxena S. Prevalence of intellectual disability: a meta-analysis of population-based studies. Res Dev Disabil 2011;32(2):419-436. [doi: 10.1016/j.ridd.2010.12.018] [Medline: 21236634]

3. Wouters M, Evenhuis HM, Hilgenkamp TIM. Physical activity levels of children and adolescents with moderate-to-severe intellectual disability. J Appl Res Intellect Disabil 2019 Jan;32(1):131-142. [doi: 10.1111/jar.12515] [Medline: 29993175]

4. Dairo YM, Collett J, Dawes H, Oskrochi GR. Physical activity levels in adults with intellectual disabilities: A systematic review. Prev Med Rep 2016 Dec;4:209-219 [FREE Full text] [doi: 10.1016/j.pmedr.2016.06.008] [Medline: 27413684]

5. Bossink L, van der Putten AA, Vlaskamp C. Understanding low levels of physical activity in people with intellectual disabilities: A systematic review to identify barriers and facilitators. Res Dev Disabil 2017 Sep;68:95-110. [doi: 10.1016/j.ridd.2017.06.008] [Medline: 28750208]

6. Temple VA. Barriers, enjoyment, and preference for physical activity among adults with intellectual disability. International Journal of Rehabilitation Research 2007;30(4):281-287. [doi: 10.1097/mrr.0b013e3282f144fb] [Medline: 17975447]

7. Balogh R, Ouellette-Kuntz H, Bourne L, Lunsky Y, Colantonio A. Organising health care services for persons with an intellectual disability. Cochrane Database Syst Rev 2008 Oct 08(4):CD007492. [doi: 10.1002/14651858.CD007492] [Medline: 18843752]

8. Haveman M, Perry J, Salvador-Carulla L, Walsh PN, Kerr M, Van Schrojenstein Lantman-de Valk H, et al. Ageing and health status in adults with intellectual disabilities: results of the European POMONA II study. J Intellect Dev Disabil 2011 Mar 14;36(1):49-60. [doi: 10.3109/13668250.2010.549464] [Medline: 21314593]

9. World Health Organization (WHO). Global recommendations on physical activity for health. World Health Organization. Geneva, Switzerland: WHO Press; 2010. URL: https://apps.who.int/iris/bitstream/handle/10665/44399/9789241599979 eng. pdf;jsessionid=D836EDD12833EBF8DDB957C7DA8113CE?sequence=1 [accessed 2020-06-13]

10. Hansen BH, Kolle E, Steene-Johannessen J, Dalene KE, Ekelund U, Anderssen SA. Monitoring population levels of physical activity and sedentary time in Norway across the lifespan. Scand J Med Sci Sports 2018 Oct 11;29(1):105-112. [doi: 10.1111/sms.13314] [Medline: 30276928]

11. Norwegian Directorate of Health. Physical activity for children, adolescents, adults, the elderly and pregnant women: national advice. Norwegian Directorate of Health. Oslo, Norway; 2019. URL: https://www.helsedirektoratet.no/faglige-rad/ fysisk-aktivitet-for-barn-unge-voksne-eldre-og-gravide [accessed 2020-06-10]

12. Melville CA, Mitchell F, Stalker K, Matthews L, McConnachie A, Murray HM, et al. Effectiveness of a walking programme to support adults with intellectual disabilities to increase physical activity: walk well cluster-randomised controlled trial. Int J Behav Nutr Phys Act 2015 Sep 29;12(1). [doi: 10.1186/s12966-015-0290-5] [Medline: 26416606]

13. van Schijndel-Speet M, Evenhuis H, van Wijck R, van Montfort KCAGM, Echteld MA. A structured physical activity and fitness programme for older adults with intellectual disabilities: results of a cluster-randomised clinical trial. J Intellect Disabil Res 2017 Jan;61(1):16-29. [doi: 10.1111/jir.12267] [Medline: 27072928]

14. Bergström H, Hagströmer M, Hagberg J, Elinder LS. A multi-component universal intervention to improve diet and physical activity among adults with intellectual disabilities in community residences: a cluster randomised controlled trial. Res Dev Disabil 2013 Nov;34(11):3847-3857 [FREE Full text] [doi: 10.1016/j.ridd.2013.07.019] [Medline: 24021393] 
15. Boer P, Moss SJ. Effect of continuous aerobic vs. interval training on selected anthropometrical, physiological and functional parameters of adults with Down syndrome. Journal of Intellectual Disability Research 2016 Jan 25;60(4):322-334. [doi: 10.1111/jir.12251] [Medline: 26805768]

16. Lante KA, Walkley JW, Gamble M, Vassos MV. An initial evaluation of a long-term, sustainable, integrated community-based physical activity program for adults with intellectual disability. Journal of Intellectual \& Developmental Disability 2011 Aug 15;36(3):197-206. [doi: 10.3109/13668250.2011.593163] [Medline: 21843034]

17. Hutzler, Korsensky O. Motivational correlates of physical activity in persons with an intellectual disability: a systematic literature review. J Intellect Disabil Res 2010 Sep;54(9):767-786. [doi: 10.1111/j.1365-2788.2010.01313.x] [Medline: 20712695]

18. Lancioni GE, Singh NN, O’Reilly MF, Sigafoos J, Alberti G, Perilli V, et al. Promoting physical activity in people with intellectual and multiple disabilities through a basic technology-aided program. J Intellect Disabil 2016 Dec 21;22(2):113-124. [doi: 10.1177/1744629516684986] [Medline: 29804524]

19. Sundblom E, Bergström H, Ellinder LS. Understanding the Implementation Process of a Multi-Component Health Promotion Intervention for Adults with Intellectual Disabilities in Sweden. J Appl Res Intellect Disabil 2015 Jul 26;28(4):296-306. [doi: 10.1111/jar.12139] [Medline: 25624120]

20. Heller T, Fisher D, Marks B, Hsieh K. Interventions to promote health: crossing networks of intellectual and developmental disabilities and aging. Disabil Health J 2014 Jan;7(1 Suppl):S24-S32. [doi: 10.1016/j.dhjo.2013.06.001] [Medline: 24456681]

21. Michalsen H, Wangberg SC, Anke A, Hartvigsen G, Jaccheri L, Arntzen C. Family members and health care workers' perspectives on motivational factors of participation in physical activity for people with intellectual disability: A qualitative study. J Intellect Disabil Res 2020 Apr 24;64(4):259-270. [doi: 10.1111/jir.12716] [Medline: 31981261]

22. de Jongh T, Gurol-Urganci I, Vodopivec-Jamsek V, Car J, Atun R. Mobile phone messaging for facilitating self-management of long-term illnesses. Cochrane Database Syst Rev 2012 Dec 12;12:CD007459 [FREE Full text] [doi: 10.1002/14651858.CD007459.pub2] [Medline: 23235644]

23. Årsand E, Muzny M, Bradway M, Muzik J, Hartvigsen G. Performance of the first combined smartwatch and smartphone diabetes diary application study. J Diabetes Sci Technol 2015 May 14;9(3):556-563 [FREE Full text] [doi: 10.1177/1932296814567708] [Medline: 25591859]

24. Chomutare T, Johansen SG, Årsand E, Hartvigsen G. Play and Learn: Developing a Social Game for Children with Diabetes. Stud Health Technol Inform 2016;226:55-58. [Medline: 27350465]

25. Taylor MJ, Taylor D, Gamboa P, Vlaev I, Darzi A. Using Motion-Sensor Games to Encourage Physical Activity for Adults with Intellectual Disability. Stud Health Technol Inform 2016;220:417-423. [Medline: 27046616]

26. Antypas K, Wangberg SC. Combining users' needs with health behavior models in designing an internet- and mobile-based intervention for physical activity in cardiac rehabilitation. JMIR Res Protoc 2014 Jan 10;3(1):e4 [FREE Full text] [doi: 10.2196/resprot.2725] [Medline: 24413185]

27. Pérez-Cruzado D, Cuesta-Vargas AI. Improving Adherence Physical Activity with a Smartphone Application Based on Adults with Intellectual Disabilities (APPCOID). BMC Public Health 2013 Dec 13;13:1173 [FREE Full text] [doi: 10.1186/1471-2458-13-1173] [Medline: 24330604]

28. Baranowski T, Blumberg F, Buday R, DeSmet A, Fiellin LE, Green CS, et al. Games for Health for Children-Current Status and Needed Research. Games Health J 2016 Feb;5(1):1-12 [FREE Full text] [doi: 10.1089/g4h.2015.0026] [Medline: 26262772]

29. Pérez-Cruzado D, Cuesta-Vargas AI. Smartphone Reminder For Physical Activity In People With Intellectual Disabilities. Int J Technol Assess Health Care 2017 Jan;33(4):442-443. [doi: 10.1017/S0266462317000630] [Medline: 28756791]

30. McConkey, Collins S. Using personal goal setting to promote the social inclusion of people with intellectual disability living in supported accommodation. J Intellect Disabil Res 2010 Feb;54(2):135-143. [doi: 10.1111/j.1365-2788.2009.01224.x] [Medline: 19874448]

31. Walker, Snell ME. Effects of augmentative and alternative communication on challenging behavior: a meta-analysis. Augment Altern Commun 2013 Jun;29(2):117-131. [doi: 10.3109/07434618.2013.785020] [Medline: 23705814]

32. Mahy J, Shields N, Taylor NF, Dodd KJ. Identifying facilitators and barriers to physical activity for adults with Down syndrome. J Intellect Disabil Res 2010 Sep;54(9):795-805. [doi: 10.1111/j.1365-2788.2010.01308.x] [Medline: 20712696]

33. Dixon-Ibarra A, Driver S, Vanderbom K, Humphries K. Understanding physical activity in the group home setting: a qualitative inquiry. Disabil Rehabil 2017 Apr 23;39(7):653-662. [doi: 10.3109/09638288.2016.1160294] [Medline: 27007887]

34. World Health Organization. World Report on Disability. Geneva, Switzerland: World Health Organization Press; 2011 Dec 13. URL: https://www.who.int/disabilities/world_report/2011/report.pdf [accessed 2020-06-13]

35. Peterson JJ. Promoting Leisure Physical Activity Participation among Adults with Intellectual Disabilities: Validation of Self-Efficacy and Social Support Scales. Journal of Applied Research in Intellectual Disabilities 2009;22(5):487-497. [doi: 10.1111/j.1468-3148.2009.00500.x]

36. Grimby G, Börjesson M, Jonsdottir IH, Schnohr P, Thelle DS, Saltin B. The "Saltin-Grimby Physical Activity Level Scale" and its application to health research. Scand J Med Sci Sports 2015 Dec;25 Suppl 4:119-125. [doi: 10.1111/sms.12611] [Medline: 26589125] 
37. Lante K, Stancliffe RJ, Bauman A, van der Ploeg HP, Jan S, Davis GM. Embedding sustainable physical activities into the everyday lives of adults with intellectual disabilities: a randomised controlled trial. BMC Public Health 2014 Oct 05;14(1):1038 [FREE Full text] [doi: 10.1186/1471-2458-14-1038] [Medline: 25284708]

38. Berg V, Haugland V, Wiik MF, Michalsen H, Anke A, Muzny M, et al. eHealth Approach for Motivating Physical Activities of People with Intellectual Disabilities. In: IFIP Advances in Information and Communication Technology, vol 573. Cham, Switzerland: Springer Nature Switzerland AG; Jan 23, 2020:31-41.

39. Kiresuk TJ, Sherman RE. Goal attainment scaling: A general method for evaluating comprehensive community mental health programs. Community Ment Health J 1968 Dec;4(6):443-453. [doi: 10.1007/BF01530764] [Medline: 24185570]

40. Jones MC, Walley RM, Leech A, Paterson M, Common S, Metcalf C. Using goal attainment scaling to evaluate a needs-led exercise programme for people with severe and profound intellectual disabilities. J Intellect Disabil 2006 Dec 21;10(4):317-335. [doi: 10.1177/1744629506070051] [Medline: 17105740$]$

41. Dijkstra A. Working mechanisms of computer-tailored health education: evidence from smoking cessation. Health Educ Res 2005 Oct;20(5):527-539. [doi: 10.1093/her/cyh014] [Medline: 15701665]

42. Palisano RJ, Rosenbaum P, Bartlett D, Livingston MH. Content validity of the expanded and revised Gross Motor Function Classification System. Dev Med Child Neurol 2008 Oct;50(10):744-750 [FREE Full text] [doi: 10.1111/j.1469-8749.2008.03089.x] [Medline: 18834387 ]

43. Jahnsen R, Aamodt G, Rosenbaum P. Gross Motor Function Classification System used in adults with cerebral palsy: agreement of self-reported versus professional rating. Dev Med Child Neurol 2006 Sep;48(9):734-738 [FREE Full text] [doi: 10.1017/S0012162206001575] [Medline: 16904019]

44. Hidecker, Paneth N, Rosenbaum PL, Kent RD, Lillie J, Eulenberg JB, et al. Developing and validating the Communication Function Classification System for individuals with cerebral palsy. Dev Med Child Neurol 2011 Aug;53(8):704-710 [FREE Full text] [doi: 10.1111/j.1469-8749.2011.03996.x] [Medline: 21707596 ]

45. Nordstrøm M, Hansen BH, Paus B, Kolset SO. Accelerometer-determined physical activity and walking capacity in persons with Down syndrome, Williams syndrome and Prader-Willi syndrome. Res Dev Disabil 2013 Dec;34(12):4395-4403 [FREE Full text] [doi: 10.1016/j.ridd.2013.09.021] [Medline: 24139709]

46. Temple VA, Stanish HI. Pedometer-measured physical activity of adults with intellectual disability: predicting weekly step counts. Am J Intellect Dev Disabil 2009 Jan;114(1):15-22. [doi: 10.1352/2009.114:15-22] [Medline: 19143459]

47. Henriksen A, Haugen Mikalsen M, Woldaregay AZ, Muzny M, Hartvigsen G, Hopstock LA, et al. Using Fitness Trackers and Smartwatches to Measure Physical Activity in Research: Analysis of Consumer Wrist-Worn Wearables. J Med Internet Res 2018 Mar 22;20(3):e110. [doi: 10.2196/jmir.9157] [Medline: 29567635]

48. Bunn JA, Navalta JW, Fountaine CJ, Reece JD. Current State of Commercial Wearable Technology in Physical Activity Monitoring 2015-2017. Int J Exerc Sci 2018;11(7):503-515 [FREE Full text] [Medline: 29541338]

49. Feehan LM, Geldman J, Sayre EC, Park C, Ezzat AM, Yoo JY, et al. Accuracy of Fitbit Devices: Systematic Review and Narrative Syntheses of Quantitative Data. JMIR Mhealth Uhealth 2018 Aug 09;6(8):e10527 [FREE Full text] [doi: 10.2196/10527] [Medline: 30093371]

50. Guralnik JM, Simonsick EM, Ferrucci L, Glynn RJ, Berkman LF, Blazer DG, et al. A short physical performance battery assessing lower extremity function: association with self-reported disability and prediction of mortality and nursing home admission. J Gerontol 1994 Mar 01;49(2):M85-M94. [doi: 10.1093/geronj/49.2.m85] [Medline: $\underline{8126356}]$

51. Aman, MG. WH Burrow, and PL Wolford, The Aberrant Behavior Checklist-Community: factor validity and effect of subject variables for adults in group homes. Am J Ment Retard 1995;100(3):283-292. [doi: 10.1007/978-1-4419-1698-3_1632] [Medline: $\underline{8554775]}$

52. Bergström H, Hochwälder J, Kottorp A, Elinder LS. Psychometric evaluation of a scale to assess satisfaction with life among people with intellectual disabilities living in community residences. J Intellect Disabil Res 2013 Mar;57(3):250-256. [doi: 10.1111/j.1365-2788.2011.01531.x] [Medline: 22369655]

53. Lee PH, Macfarlane DJ, Lam TH, Stewart SM. Validity of the International Physical Activity Questionnaire Short Form (IPAQ-SF): a systematic review. Int J Behav Nutr Phys Act 2011 Oct 21;8:115 [FREE Full text] [doi: 10.1186/1479-5868-8-115] [Medline: 22018588]

54. Bailey KV, Ferro-Luzzi A. Use of body mass index of adults in assessing individual and community nutritional status. Bulletin of the World Health Organization 1995;73(5):673. [Medline: 8846494]

55. Freire AN, Guerra RO, Alvarado B, Guralnik JM, Zunzunegui MV. Validity and reliability of the short physical performance battery in two diverse older adult populations in Quebec and Brazil. J Aging Health 2012 Aug;24(5):863-878. [doi: 10.1177/0898264312438551] [Medline: 22422762]

56. Bergland, Strand BH. Norwegian reference values for the Short Physical Performance Battery (SPPB): the Troms $\emptyset$ Study. BMC Geriatr 2019 Aug 08;19(1):216 [FREE Full text] [doi: 10.1186/s12877-019-1234-8] [Medline: $\underline{31395008]}$

57. Olsen CF, Bergland A. Reliability of the Norwegian version of the short physical performance battery in older people with and without dementia. BMC Geriatr 2017 Jun 09;17(1):124 [FREE Full text] [doi: 10.1186/s12877-017-0514-4] [Medline: 28599623]

58. Oppewal A, Festen DAM, Hilgenkamp TIM. Gait Characteristics of Adults With Intellectual Disability. Am J Intellect Dev Disabil 2018 May;123(3):283-299. [doi: 10.1352/1944-7558-123.3.283] [Medline: 29671638] 
59. Torres-Unda J, Polo V, Dunabeitia I, Bidaurrazaga-Letona I, García-Gil M, Rodriguez-Larrad A, et al. The Feldenkrais Method improves functioning and body balance in people with intellectual disability in supported employment: A randomized clinical trial. Res Dev Disabil 2017 Nov;70:104-112. [doi: 10.1016/j.ridd.2017.08.012] [Medline: 28923375]

60. Halvorsen M, Aman MG, Mathiassen B, Brøndbo PH, Steinsvik OO, Martinussen M. Psychometric Properties of the Norwegian Aberrant Behavior Checklist and Diagnostic Relationships in a Neuro-Pediatric Sample. Journal of Mental Health Research in Intellectual Disabilities 2019 Jul 04;12(3-4):234-255 [FREE Full text] [doi:

10.1080/19315864.2019.1630872]

61. Guillemin F, Bombardier C, Beaton D. Cross-cultural adaptation of health-related quality of life measures: Literature review and proposed guidelines. Journal of Clinical Epidemiology 1993 Dec;46(12):1417-1432. [doi: 10.1016/0895-4356(93)90142-n] [Medline: 8263569]

62. Turner-Stokes L. Goal attainment scaling (GAS) in rehabilitation: a practical guide. Clin Rehabil 2009 Apr;23(4):362-370. [doi: 10.1177/0269215508101742] [Medline: 19179355]

63. Krasny-Pacini A, Hiebel J, Pauly F, Godon S, Chevignard M. Goal attainment scaling in rehabilitation: a literature-based update. Ann Phys Rehabil Med 2013 Apr;56(3):212-230 [FREE Full text] [doi: 10.1016/j.rehab.2013.02.002] [Medline: 23562111]

64. Willis C, Nyquist A, Jahnsen R, Elliott C, Ullenhag A. Enabling physical activity participation for children and youth with disabilities following a goal-directed, family-centred intervention. Res Dev Disabil 2018 Jun;77:30-39. [doi:

10.1016/j.ridd.2018.03.010] [Medline: 29635224]

65. Estival S, Krasny-Pacini A, Laurier V, Maugard C, Thuilleaux D, Postal V. Cognitive Training Targeting Planning Dysfunction in Adults with Prader-Willi Syndrome: Brief Report of a Study Protocol. Dev Neurorehabil 2019 Nov 29;22(8):569-575. [doi: 10.1080/17518423.2019.1642414] [Medline: 31355692]

66. Elinder LS, Bergström H, Hagberg J, Wihlman U, Hagströmer M. Promoting a healthy diet and physical activity in adults with intellectual disabilities living in community residences: design and evaluation of a cluster-randomized intervention. BMC Public Health 2010 Dec 13;10(1):761 [FREE Full text] [doi: 10.1186/1471-2458-10-761] [Medline: 21144033]

67. Rimmer JH, Heller T, Wang E, Valerio I. Improvements in Physical Fitness in Adults With Down Syndrome. Am J Mental Retard 2004;109(2):165. [doi: 10.1352/0895-8017(2004)109<165:iipfia>2.0.c0;2]

68. Shields N, Taylor NF, Wee E, Wollersheim D, O'Shea SD, Fernhall B. A community-based strength training programme increases muscle strength and physical activity in young people with Down syndrome: a randomised controlled trial. Res Dev Disabil 2013 Dec;34(12):4385-4394. [doi: 10.1016/j.ridd.2013.09.022] [Medline: 24120754]

69. Oppewal A, Hilgenkamp TIM. Adding meaning to physical fitness test results in individuals with intellectual disabilities. Disabil Rehabil 2020 May 02;42(10):1406-1413. [doi: 10.1080/09638288.2018.1527399] [Medline: 30712416]

\author{
Abbreviations \\ ABC: Aberrant Behavior Checklist \\ ABC-C: Aberrant Behavior Checklist-Community \\ ID: intellectual disability \\ mHealth: mobile health \\ PA: physical activity \\ SPPB: Short Physical Performance Battery \\ WHO: World Health Organization
}

Edited by G Eysenbach; submitted 08.04.20; peer-reviewed by C Forbes, T Mettler, K Ng; comments to author 08.05.20; revised
version received 19.05.20; accepted 19.05.20; published 29.06.20
Please cite as:
Michalsen H, Wangberg SC, Hartvigsen G, Jaccheri L, Muzny M, Henriksen A, Olsen MI, Thrane G, Jahnsen RB, Pettersen G, Arntzen
C, Anke A
Physical Activity With Tailored mHealth Support for Individuals With Intellectual Disabilities: Protocol for a Randomized Controlled
Trial
JMIR Res Protoc $2020 ; 9(6):$ e19213
URL: http://www.researchprotocols.org/2020/6/e19213/
doi: $10.2196 / 19213$
PMID: $\underline{32437328}$

CHenriette Michalsen, Silje Camilla Wangberg, Gunnar Hartvigsen, Letizia Jaccheri, Miroslav Muzny, André Henriksen, Monica Isabel Olsen, Gyrd Thrane, Reidun Birgitta Jahnsen, Gunn Pettersen, Cathrine Arntzen, Audny Anke. Originally published in 
JMIR Research Protocols (http://www.researchprotocols.org), 29.06.2020. This is an open-access article distributed under the terms of the Creative Commons Attribution License (https://creativecommons.org/licenses/by/4.0/), which permits unrestricted use, distribution, and reproduction in any medium, provided the original work, first published in JMIR Research Protocols, is properly cited. The complete bibliographic information, a link to the original publication on http://www.researchprotocols.org, as well as this copyright and license information must be included. 